HPV identification in vaginal swabs and nasopharyngeal aspirates

\begin{tabular}{llllll}
\hline & $H P V$ positive & HPV 6/11 & HPV 16/18 & HPV 31/33 & Unidentified \\
\hline Vaginal swabs & $15 / 124$ & 2 & 1 & 2 & 10 \\
$\begin{array}{l}(12 \cdot 1 \%) \\
\text { Nasopharyngeal }\end{array}$ & $\begin{array}{l}10 / 109 \\
\text { aspirates }\end{array}$ & 2 & 1 & 1 & $(66 \cdot 6 \%)$ \\
\hline
\end{tabular}

demonstrated in $6 / 47(12 \cdot 7 \%)$ vaginal swabs and in $4 / 47(8.5 \%)$ nasopharyngeal aspirates. In two cases both the mother and the neonate were HPV positive. One pair was concordant for HPV6/11 infection and the other was putatively concordant for unidentified HPV. In two cases the neonate was HPV positive (one HPV6/11 and one unidentified HPV) and the mother HPV negative and in four cases the mother was HPV positive (one HPV16/18 and three unidentified HPV) and the neonate negative. Our result is consistent with published data and again confirms that a substantial fraction of cytologically normal women is infected with HPV. The results of HPV typing in our female population may appear surprising. Our probes did not identify the HPV type in more than $60 \%$ of samples positive to the generic probe. This data seems to be in contrast with most published data according to which HPV 16 is the most frequent HPV type in cervical samples from apparently healthy women. ${ }^{4}$ However, our results also seem to be confirmed by data obtained at the Department of Pathology of Siena University where HPV amplification and typing is routinely carried out on cervical biopsies referred for histological evaluation. According to them more than $50 \%$ of HPV samples positive after amplification with MY09/MY11 primers were negative to type-specific PCR (R Santopietro, personal communication). It is therefore possible that HPV 16 is the most common HPV type among the most common genital HPV types but it is also likely that most amplified HPV will fall into the "unidentified" category, as already proposed by Schiffmann and Gravitt, when general-primers PCR protocols are used. ${ }^{5}$ In conclusion our results confirm previous observations on the frequency of HPV infection in apparently healthy women and show that these viruses can be transmitted vertically during delivery. ${ }^{6}$ Since this event may be the cause of laryngeal papillomatosis it is a matter of debate whether or not to perform a cesarean section in infected women. To date there are insufficient data to advocate the need of cesarean section in infected women ${ }^{7}$ and this position should be held until further studies will clarify the determinants of laryngeal papillomatosis. Our data also show that singlepoint sampling, which would have been useful for screening in clinical practice, is not a reliable method to assess the risk of vertical transmission of HPV for which multiple sampling in mothers and children is required.

C MAZZATENTA

M FIMIANI

L ANDREASSI

Department of Dermatology of Siena University P BUFFI

Unit of Gynaecology

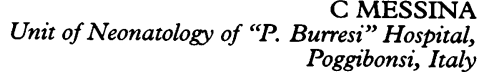

Correspondence to: Prof Michele Fimiani, Department of Dermatology of Siena University, Policlinico Le Scotte, Viale Bracci, 53100 Siena, Italy.

1 Oriel JD. Sexually transmitted diseases in children: human papillomavirus infection. Genitourin Med 1992;68:80-3

2 Sedlacek TV, Lindheim S, Eder C, Hasty L, Woodland M,

Ludomirsky A, et al. Mechanism for human papilloLudomirsky A, et al. Mechanism for human papillo1989;161:55-9.

3 Bauer HM, Ting Y, Greer CE, Chambers JC, Tashiro CJ, Chimera J, et al. Genital human papillomavirus infection in female university students as determined by a PCR based method. $\mathcal{F A M A}$ 1991;265:472-7.

4 Melchers WGJ, van de Brule AJ, Walboomers JMM, De Bruin $M$, Burger $M$, Herbrink $P$, et al. Increased detection rate of human papillomavirus in cervical scrapes by the polymerase chain reaction as compared to modified FISH and Southern blot analysis. $f$ Med Virol 1989;27:329-35.

5 Shiffman MH. Epidemiology of cervical human papillomavirus infections. In: H zur Hausen (ed). Human Pathogenic Papillomaviruses. Springer Verlag, Heidelberg, 1994:55-81.

6 Pakarian F, Kaye J, Cason J, Kell B, Jewers R, Devias NW et al. Cancer associated human papillomaviruses: perinatal transmission and persistence. $\mathrm{Br} \mathcal{F}$ Obstet Gynaecol 1994;101:514-7.

7 Center for Disease Control. 1989 sexually transmitted diseases treatment guidelines. $M M W R \quad 1989 ; 38$ (suppl): $18-21$.

Accepted for publication 7 August 1996.

\section{Cytomegalovirus retinitis in a healthy antiretroviral naive HIV positive male with a CD4 count of $277 / \mathrm{mm}^{3}$}

Cytomegalovirus (CMV) retinitis is a common sight threatening complication of late HIV disease, occurring rarely with $\mathrm{CD}^{+}$lymphocyte counts above $100 / \mathrm{mm}^{3}$ and most frequently when the $\mathrm{CD}^{+}$lymphocyte count falls below $50 / \mathrm{mm}^{3} .^{1}$ Four cases of CMV retinitis occurring in HIV patients with CD4 counts greater than $200 / \mathrm{mm}^{3}$ have been described. In three of these cases, the CD4 counts were $255 / \mathrm{mm}^{3}$ and $235 / \mathrm{mm}^{3}$ and $355 / \mathrm{mm}^{3}$ at the time of CMV diagnosis. ${ }^{23}$ However, these patients were receiving zidovudine antiretroviral therapy which may complicate interpretation of CD4 counts. As demonstrated by Concorde, ${ }^{4}$ zidovudine may sustain CD4 counts without translating into clinical benefit. Although the reason for this is unclear, one suggestion was that zidovudine may alter the proportion of poorly functioning CD4 cells in the peripheral blood. In the fourth case, the patient had a CD4 count of $366 / \mathrm{mm}^{3}$ and although he had not previously had antiretroviral therapy, his CD4 count and immune function may have been altered by a previous splenectomy. ${ }^{4}$

We report a case of CMV retinitis occurring in a 42 year old HIV seropositive homosexual man, who was antiretroviral naive, in good health with no prior AIDS defining diagnosis and no other immunocompromising condition. He presented with a history of "floaters" preceding the development of a shadow in the vision of his right eye. He had a relatively stable 
CD4 lymphocyte count which was $277 / \mathrm{mm}^{3}$ at the time of diagnosis. He was evaluated by two ophthalmologists, both of whom confirmed CMV retinitis with a retinal detachment. In addition he had a vitreal biopsy which was positive by PCR for CMV and negative for herpes simplex, herpes zoster and Toxoplasma gondii. His retinitis failed to respond to intravenous genciclovir, but subsequently responded to induction with 3 weeks of intravenous foscarnet therapy and remains quiescent on IV foscarnet maintenance therapy. Following diagnosis of CMV infection his CD4 count fell to $90 / \mathrm{mm}^{3}$ within 6 months, although it improved with combination antiretroviral therapy (zidovudine and zalcitabine). Interestingly, in two of the other non-splenectomised patients CD4 counts fell precipitously soon after diagnosis (from $255 / \mathrm{mm}^{3}$ to $15 / \mathrm{mm}^{3}$ over 8 months in one patient and $235 / \mathrm{mm}^{3}$ to $32 / \mathrm{mm}^{3}$ over 7 months in the other) reinforcing the importance of aggressive antiretroviral therapy once an opportunistic infection associated with immunocompromise develops.

A non HIV related case with a normal CD4 count has highlighted the importance of CD4 cell function in the prevention of CMV retinal infection. ${ }^{5}$ HIV infection causes a great heterogeneity of immunological dysfunction. The CD4 count acts as a surrogate marker for the level of immune dysfunction but may hide functional as well as other subtle abnormalities of the immune system. CD4 counts are useful for predicting patients at risk of CMV retinitis and, therefore, those who may benefit from screening. However, although rare, this case further demonstrates that CMV retinitis can occur at CD4 counts greater than $200 / \mathrm{mm}^{3}$ and should serve to caution HIV physicians that the diagnosis of CMV retinitis is not excluded by a relatively high CD4 count.

I FAIRIEY $M$ A WAUGH Department of Genitourinary Medicine, General Infirmary at Leeds,

1 Pertel P, Hirschtick R, Phair J, Chmiel J, Poggensee L, Murphy R. Risk of developing Cytomegalovirus retinitis in persons infected with Human Immunodeficiency Virus. F Acquir Immune Defic Syndr 1992;5:1069-74.

2 Fekrat S, Dunn JP, Lee D, Miller T, Jabs DA. Cytomegalovirus retinitis in HIV-infected patients with elevated CD4 counts. Arch Ophthalmol 1995;113:18.

3 Baglivo E, Dosso A, Leuenberger PM, Jelk L. Cytomegalovirus retinitis in an AIDS patient without severe depletion in CD4 cell count [letter]. B $f$ severe depletion in CD4
Ophthalmol 1995;79:962-3.

4 Concorde coordinating committee. Concorde: MRC/ ANRS randomised double-blind controlled trial of immediate and deferred zidovudine in symptom-free HIV infection. Lancet 1994;343:871-80.

5 Angus BJ, Green ST, McKinley JJ. McMenamin J, Walker E. HIV-associated CMV retinitis occurring in the setting of a high CD4 count: what about AIDS definitions? Int STD AIDS 1994;5:71-2.

6 Rahhal FM, Rosberg DF, Polsky B, Thoron L, Heinemann M. Cytomegalovirus retinitis in patient with a normal Thelper (CD4) count. Arch Ophthalmol 1993;111:325-6.

Accepted for publication 4 September 1996

\section{Acute cytomegalovirus prostatitis in AIDS}

Genitourinary tract disorders are common in the acquired immunodeficiency syndrome (AIDS) including a wide spectrum of abnor- malities due to an AIDS-related neoplasm or infection due to typical microorganisms or opportunistic pathogens. ${ }^{1}$

Recent papers have emphasised the importance of prostatic disease as an emerging problem for patients with HIV infection. Indeed, involvement of prostatic gland is becoming more prevalent in patients with AIDS than in the general population. ${ }^{2}$

Several clinical and pathologic surveys have described cases of prostatic disease caused by typical bacteria, including Escherichia coli and other Gram-negative bacteria, Staphylococcus aureus, Haemophilus parainfluenzae and Salmonella typhi, Mycobacterium tuberculosis and atypical mycobacteria, and fungal pathogens such as Histoplasma capsulatum and Cryptococcus neoformans. ${ }^{2}$

HIV itself alone, in the absence of other infecting agents, also has been implicated as a cause of prostatic abnormalities. ${ }^{2}$

In the current literature we rarely find reports of prostatitis caused by cytomegalovirus (CMV) in HIV-positive persons. ${ }^{1}$

We here report a rare case of prostatitis due to CMV infection in a patient with AIDS in whom the diagnosis was established after death and who received antiviral chemotherapy with ganciclovir.

A 34 year old intravenous drug abuser with AIDS was hospitalised complaining of lower abdominal pain, urinary frequency without dysuria, and fever. Six months before he had came to our attention because of CMV retinitis that had been treated with ganciclovir with a successful response, but the patient had not continued with a maintenance therapy. At the moment of the admission he appeared severely ill. The laboratory studies revealed a $\mathrm{CD} 4+$ lymphocyte cells count below $50 / \mu \mathrm{l}$, a white blood cells count (WBC) of $3400 / \mathrm{mm}^{3}$ with $60 \%$ neutrophils, and $18 \%$ lymphocytes, an erythrocyte sedimentation (ESR) rate of 78 $\mathrm{mm}$ /hour, and a lactate dehydrogenase $(\mathrm{LDH})$ value of $650 \mathrm{U} / 1$. Urinanalysis revealed one to five WBC per high power field without casts and trace amounts of protein. Repeated urine culture grew CMV. Microscopic and cultural examinations of blood, sputum and stool specimens were unremarkable. An ultrasound evaluation of the lower abdomen was normal. The examination of the prostate was normal. An active CMV retinitis in the right ocular fundus was detected. Reinduction therapy with ganciclovir was started in association with cotrimoxazole. The patient's clinical condition continued to deteriorate, and he died after two weeks of hospitalisation. Post mortem examination reported a disseminated CMV infection, with prominent localisation in the prostatic gland, the lungs and the adrenal glands. Macroscopic evaluation revealed a normal sized prostate, while microscopically there was evidence of a large inflamed prostatic epythelium with multiple areas of tissue necrosis containing CMV intranuclear and intracytoplasmatic inclusions.

Although infection with CMV is commonly benign in the immunocompetent person, the virus remains a major cause of morbidity and 